\title{
Tracking and Predicting End-to-End Quality in Wireless Community Networks
}

\author{
Pere Millan, Carlos Molina \\ Dept. of Computer Engineering \\ Universitat Rovira i Virgili \\ Tarragona, Spain \\ \{pere.millan,carlos.molina\}@urv.net
}

\author{
Emmanouil Dimogerontakis \\ Leandro Navarro, Roc Meseguer \\ Dept. of Computer Architecture \\ Universitat Politecnica de Catalunya \\ Barcelona, Spain
}

\author{
Bart Braem, Chris Blondia \\ Dept. of Mathematics and Computer Science \\ iMinds - MOSAIC - University of Antwerp \\ Antwerpen, Belgium \\ \{bart.braem,chris.blondia\}@uantwerpen.be
}

\{edimoger,leandro,meseguer\}@ ac.upc.edu

\begin{abstract}
Community networks are an emergent model with mottos like "a free net for everyone is possible" or "don't buy the network, be the network". Their social impact is measurable, as the community is provided with the right and opportunity of communication. The combination of wired and wireless links in these networks, and the unreliable nature of the wireless medium, poses several challenges to the routing protocol. End-toEnd quality tracking helps the routing layer to select paths that maximize the delivery rate and minimize traffic congestion. We believe that End-to-End quality prediction can be a technique that surpasses End-to-End quality tracking by foreseeing which paths are more likely to change quality. In this work, we focus on End-to-End quality prediction by means of time-series analysis. We apply this prediction technique in the routing layer of largescale, distributed, and decentralized networks. We demonstrate that it is possible to accurately predict End-to-End Quality with an average Mean Absolute Error of just 2.4\%. Particularly, we analyze the path properties and path ETX behavior to identify the best prediction algorithm. Moreover, we analyze the EtEQ prediction accuracy some steps ahead in the future and also its dependency of the time of the day.
\end{abstract}

Keywords-Community Networks, End-to-End Quality Prediction, Time-Series Analysis

\section{INTRODUCTION}

Community networks are large-scale, distributed, and decentralized networking infrastructures with several nodes, links, and services. This kind of networks are extremely diverse and dynamic, because of their decentralized nodes, their mix of wired and wireless technologies, their several routing schemes, and their diverse services and applications [1]. Those networks are made available to a community of people living in the same area. The network management is based on an open peering agreement, which avoids barriers for the network participation. Ownership, governance, and knowledge are also open (community members own and manage these networks). Community networks (i.e., FunkFeuer [2] and Guifi.net [3]) grow dynamically with regards to the number of links.

Community Networks features (large, heterogeneous, dynamic, decentralized) raise challenges of interest for researchers [4]. One of the most important challenges is the effect of the asymmetrical features and unreliability of wireless communications on network performance and routing protocols. Many metric-based routing protocols for mesh networks that track Link Quality (LQ) and select higherquality links have been proposed to minimize traffic congestion and maximize delivery rate [5], [6], [7], [8]. Hence, when routing packets through an unreliable network, LQ tracking is definitely a key method to apply. Moreover, routing algorithms should avoid weak links as soon as possible [9], and whenever possible [10]. LQ estimation [11] (or prediction [12]) approach increases the improvements achieved by LQ tracking in routing performance. Usually, real-time metrics do not provide enough information to detect the degradation or activation of a link at the right moment. Therefore, prediction is needed to foresee LQ changes and take the appropriate measures.

End-to-End Quality (EtEQ) or Path Quality extends the LQ concept to the full communication-path (between sender and receiver) and it is computed based on the quality (ETX) of the individual links that conform the communication path. In this paper, we want to analyze if our previous work on LQ [13] is applicable to the full communication path (EtEQ tracking and prediction) and determine what differences exist between individual LQ and EtEQ. To the best of our knowledge, no previous work explores EtEQ prediction in the routing layer of large-scale, distributed, and decentralized systems.

The main contributions of this work are the following: (1) A detailed analysis of path properties and path ETX behaviour in Wireless Mesh Community Networks (WMCN), showing that path quality prediction is possible and meaningful. (2) The use of Time Series analysis to estimate EtEQ in the routing layer for real-world WMCN. (3) Clear evidence that EtEQ values computed through Time Series algorithms can make accurate predictions in $\mathrm{WMCN}$. And (4) A detailed analysis of the prediction accuracy for the next step considering also the time of the day and for some steps ahead in the future.

This paper is structured as follows. Section II provides the necessary background, while section III presents our data set analysis and introduces our proposal by showing the EtEQ behaviour of a WMCN. Section IV overviews prediction in computer networks focused on link/path quality prediction in WMCN. Section V describes the experiments made to show the value of EtEQ prediction followed by results analysis. Finally, Section VI provides some conclusions and future work. 


\section{BACKGROUND}

It is well known that selecting high quality links in realworld networks composed by wireless channels with unpredictable conditions is a big challenge for achieving high delivery rate and performance. Our research goal in this paper is to assess if the improvements previously achieved by applying LQ tracking and prediction techniques, are also achievable when considering the full communication path (End-to-End Quality). To evaluate the potential benefits of this proposal, we first analyze the characteristics of a well known, free and experimental WMCN that deals with the Optimized Link State Routing (OLSR) protocol to maintain the network topology.

FunkFeuer [2] is a non-commercial project maintained by computer enthusiasts that install Wi-Fi antennas across rooftops in several places of Austria that are relatively close to each other (Vienna, Graz, Weinviertel and Bad Ischl). Currently, there are around 2000 wired and wireless links and every week new antennas are added to the network. FunkFeuer uses the OLSR-NG routing protocol, which expands the capabilities of the OLSR protocol and makes it highly scalable. In fact, some members of the FunkFeuer network are actively involved in the olsr.org open source project as developers, testing the protocol in the network. Furthermore, the FunkFeuer network maintains open data sets, available also through the CONFINE Project open data platform [14], which were used in this work. The chosen data set is composed of OLSR information such as routing tables and network topology data, collected during 7 days in the period from April 28th to May 4th, 2014. The largest of the shortest paths in the network (diameter) is 18. This means that there are several paths where packets have to go through a relatively high number of Hops in order to reach their destination. The routing protocol must, therefore, react quickly to any change in the network topology since this will be critical to achieve high performance.

As stated before, FunkFeuer assumes OLSR, a link-state routing protocol where every node maintains a connectivity map for all the network. Exploiting this OLSR property, FunkFeuer publishes its complete network information from the point of view of a single node (ego-network). While convenient for data collection, this method comes with the downside that the data set is biased and does not represent the real network state, since the time for event propagation throughout the network is not negligible. In other words, the higher the distance between a node and an event that happens in the network, the later this event will be present in the nodes global view. Therefore, prediction of path changes can improve local node routing decisions, since it can provide the node with an estimation about the future local and remote events.

ETX [15] is an active-probing link metric, designed for MANETs and widely used in mesh protocols, based on estimating the bidirectional loss ratios of a link. The ETX value of a link is the number of expected transmissions needed to send a packet over the link and is calculated as follows: $E T X=1 /(L Q * N L Q)$, where LQ and NLQ stand for the "Link Quality" and the "Neighbor Link Quality" of that link, respectively. The ETX of a path is defined as the sum of the ETX value of the links that form the path. As a result, ETX is always greater or equal to the actual number of Hops in the path. The difference between the path ETX and the number of Hops of the path is the expected number of losses.

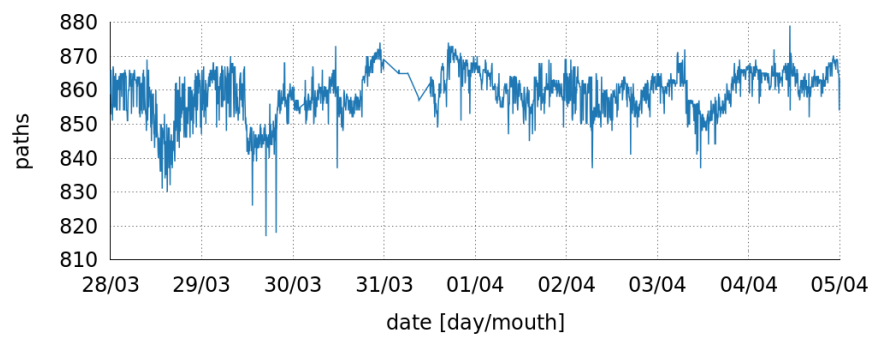

Figure 1. Temporal evolution of the number of paths.

The OLSR protocol uses ETX to choose, for each device and packet, the next hop. Concerning physical links, the LQ assumed by OLSR is defined as the fraction of successful packets (HELLO) that were received by a node from a given neighbor within a certain time window, while the NLQ is the fraction of successful packets that were received by the neighbor within a time period. Concerning paths, OLSR calculates the ETX of all the possible paths from the source to the destination, as described above, and chooses the one with minimum ETX value. That is to say, the ultimate decision to be made by OLSR will be about the selected paths; therefore, the final metric value that will be the subject of comparison will relate to the whole path. As a result, prediction of the path ETX will allow more efficient routing decisions in an unstable environment, taking also into account the ego-network measurement effect explained previously. It is important to point out here that LQ as defined by ETX and studied in this work ignores the parameters of transmitted-packets size as well as link transmission rate. Consequently, this work considers that the significant path quality parameter is packet loss.

\section{Data Set Analysis}

In this section we present the performed data set analysis, that provided us with a better insight into ETX and path behaviour, but also helped us confirm our hypotheses that path quality prediction is possible and meaningful.

\section{A. Path Behavior}

Our first study analyses the temporal evolution of the number of paths (routing entries). Figure 1 shows this evolution in a period of 8 days. We can observe that the fluctuation of the number of paths seen by one node is quite significant (about 7\% of variation). This observation contrasts with the general assumption in mesh networks that the number of paths is stable. Multi-Point Relay selection, that is normally made by consensus, does not take into account this observed variation and, therefore, the accuracy of finding the best routes can be compromised. Our time series based analysis will consider this variation to make accurate predictions of future EtEQ.

Figure 2 plots the fraction of snapshots over the total for which the path has been present, namely the persistence of each path. Considering that the snapshots were taken every 5 minutes, we observe that about $10 \%$ of paths lie below $60 \%$ of persistence, but the remaining $90 \%$ of paths are very stable. We notice that the $10 \%$ of unstable paths can explain the variations in Figure 1. Although the amount of unstable paths is significant, a path with very low persistence cannot 


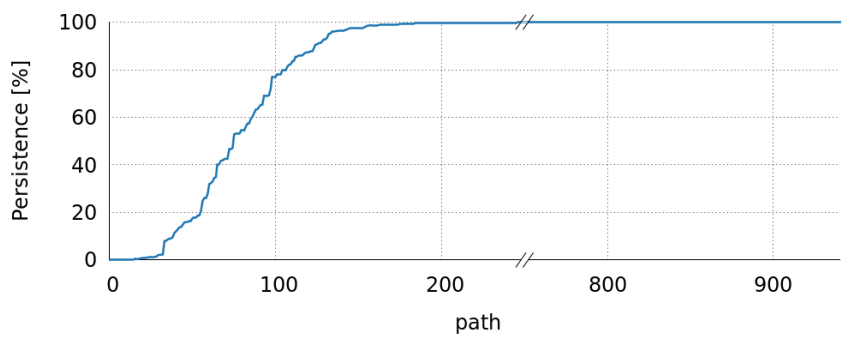

Figure 2. Persistence of paths.

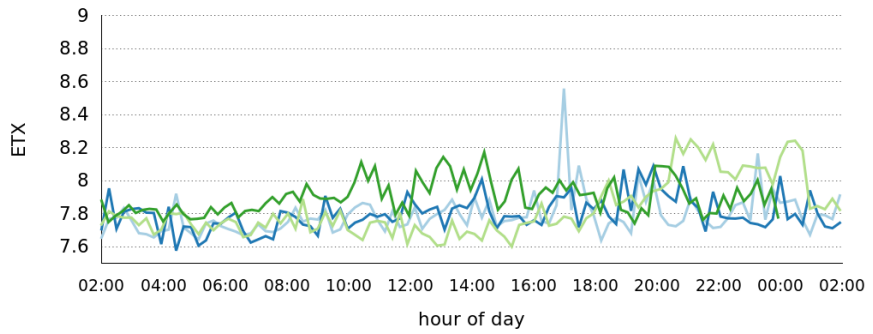

Figure 3. Temporal evolution of the average ETX of paths.

be useful for predicting the behavior of stable paths. Therefore, taking into account that the data set represents 8 days, for the analysis done in the following sections we ignore paths that are stable less than 2 days in total (persistence below 25\%).

\section{B. ETX Behavior}

To understand the behavior of ETX path quality, we made some additional measurements. Notice that in the data set introduced above, the corresponding EtEQ is ETX path quality.

Figure 3 shows the evolution of average ETX path quality inside a day for the 5 working days included in the data set. From this figure, it can be seen that ETX path values are more stable at night, between 2 a.m. and 8 a.m. Throughout the day, between 9 a.m. and 1 a.m., there are more fluctuations, reaching differences of even 1 expected transmission. Variation of actual ETX path values can be significantly higher, but this information is hidden by the average value. For non-working days this behavior is less apparent. Similar patterns are often found in network usage data sets, therefore we can assume that the reasons for the depicted behavior is the variation of network traffic and interference during the day, leading to packet loss. This observation suggests that we possibly have to expect different prediction accuracy depending the day and time when prediction is applied.

To analyze the relation between path Hop count and its corresponding ETX, Figure 4 plots the frequency of each of these values. We can observe that Hops and ETX overlap between the values 1 and 4 . That means that the quality of short paths is maximum. However, for values 5 or higher, ETX path values present deviations that can be translated as decreased EtEQ and a large number of needed packet retransmissions. Notice that the more frequent values of Hops and ETX (more than 5\% each) are in the range from 5 to 10.

In our last study, we analyze the dispersion of ETX according to the number of Hops. In this way, Figure 5 contains

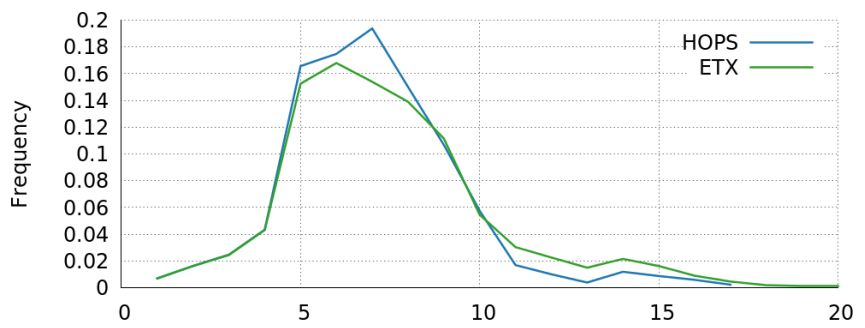

Figure 4. Frequency of path lengths based on hop count and ETX.

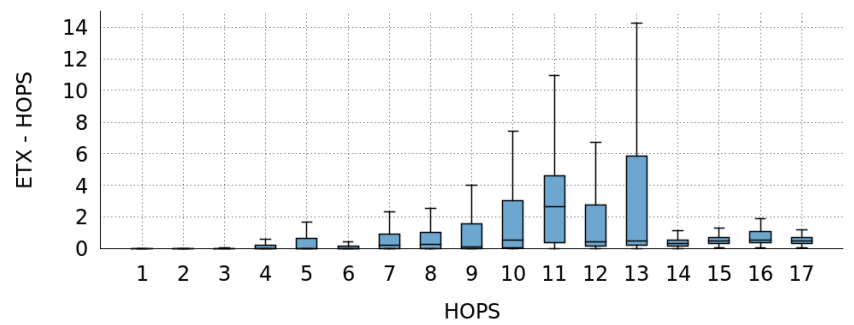

Figure 5. Distribution of ETX of paths versus the number of Hops, normalized by corresponding number of Hops.

the boxplots of ETX, for individual number of Hops. The ETX values are normalized with the corresponding number of Hops to provide a better idea of the scale of the deviation, taking into account that the ETX deviation is not correlated with the number of Hops. We can observe that ETX dispersion is minimal for range 1 to 4 Hops, it is significant for range 5 to 9 Hops, and relevant for 10 to 13 Hops. For 14 Hops and above, ETX dispersion is minimal again. Therefore, we can conclude that the prediction would be more or less accurate depending on the number of Hops.

From the above preliminary observations and data-set studies we reached the conclusions that from a node's ego-network point-of-view there is a non-negligible amount of unstable paths for which the ETX path value shows a significant deviation from the optimal value. Therefore, investigating the EtEQ behavior as well as finding efficient EtEQ prediction strategies based on time-series analysis will result to valuable input for routing in $\mathrm{WMCN}$.

\section{Link Quality PRediction In COMmunity NETWORKS}

Prediction techniques have been applied in several ways such as routing traffic reduction [16], [17], [18], energy efficient routing [19], [20], [21] or LQ estimation.

LQ tracking has been previously applied in several scenarios in different ways [5], [6], [7], [8] to select higher quality links that maximize delivery rate and minimize traffic congestion. LQE (Link Quality Estimators) [11], [12] are in charge of measuring the quality of the links between nodes based on physical or logical metrics. Physical metrics focus on the received signal quality and logical metrics focus on the percentage of lost packets. LQE with metrics like LQI (Link Quality Indication) [22], SNR (Signal-to-Noise Ratio) [23] or RSSI (Received Signal Strength Indication) [24] fit in the former category, whereas metrics like RNP (Required Number 
of Packets) [25], ETX (Expected Transmission Count) [15], [25] or PSR (Packet Success Rate) [5] fit in the latter. All these metrics can be used by LQE in isolation or as a combination of some of them [11], [12], [27] to select the more suitable neighbor nodes when making routing decisions. LQ prediction is used in addition to LQ tracking to determine beforehand which links are more likely to change their behavior. Although LQ prediction is not identical with EtEQ prediction some of the above techniques can be very similar [5], [15], [22], [23], [25], [27]. This relation is even more direct in the case of ETX EtEQ, studied in this paper, which is a linear function of the ETX LQ. As a result, the routing layer can take better decisions at the appropriate moment.

End-to-End Quality has not been widely considered in the past. In fact, the main efforts have been focused on determining the cost of a single link and then extended to the path by assuming the cost of a path as the sum of costs of several links. For instance, MARA [28] has been proposed as a method to make more accurate routing decisions by combining route quality evaluation and automatic rate selection. To do this, MARA computes ETX of every link at every available rate, estimating metrics as SNR, packet error rate, and probe packet size. End-to-End Retransmissions (EER) operation model [29] claims that the total cost of a path cannot be expressed as a linear sum of individual link costs. Therefore, variations of this simple formulation as incorporating error rate in the link cost are proposed. This led the authors to achieve significant energy savings compared to traditional minimum energy approaches. ETOP [30] has been proposed as a path metric to determine reliable end-to-end packet delivery. In the same way as EER, this work assumes that the cost of a path does not only rely on the quality of individual links but also on their relative position on the path. Finally, EED/WEED [31] is another approach that was designed as a link/path metric to select paths with minimum end-to-end delay and high network throughput but considering load balancing of routing. In any case, there is no work concerning prediction of path quality in a Wireless Mesh Community Network.

There are some relevant works that must be paid special attention as they are related to our study: Wang, et al [32], Maccari and Cigno [32] and Millán et al [13]. Wang et al [32] introduces the MetricMap mechanism, that is is fundamentally a routing protocol for wireless sensor networks that uses a learning-enabled method for LQ assessment. Based on the observation that high traffic rates make tracking link qualities more difficult, this protocol uses prediction methods to estimate them in advance. In a first stage, a machinelearning algorithm is applied to classify link qualities. Two types of classifiers are evaluated: a decision tree and a rulebased classifier. The data used to train both classifiers was preclassified offline based on a LQ indicator and other metrics that represent some features of the nodes. In a second stage, the MetricMap routing protocol estimates the LQ at runtime by replacing the current traffic information with the rules collected offline from the classifiers. Results show that MetricMap can achieve a significant improvement on the data delivery rate in high traffic rate applications.

Maccari and Lo Cigno [33] have considered the FunkFeuer network focusing on link layer properties, topological patterns and routing performance. They have analyzed the quality of the routes and proposed a couple of techniques to select the MultiPoint Relay (MPR) nodes in the Optimized Link State Routing (OLSR) protocol. Traditionally, routing algorithms assume that mesh networks are fairly stable but we have also observed that this is not completely true. Therefore, MPR selection should consider the path variability of a node instead of selecting them by agreement. We also analyze the quality of routes but focused on estimating its future quality, to improve the routing layer to select links that maximize the delivery rate and minimize traffic congestion.

Finally, Millán et al [13] analyze the behavior of link quality prediction in the routing layer of large-scale, distributed and decentralized systems composed of many nodes, links, content and services. In summary, the main contributions of this work are (1) the employ of time series analysis to estimate link quality in the routing layer for real-world WMCN, (2) the detailed evaluation of results, assuming several learning algorithms to show the potentiality of time series analysis for estimating link quality in short and long term and (3) the evidence that link quality computed from time series can be used to accurately predict future values in WMCN. This work is the most similar to ours as both deal with time series analysis to improve the routing protocol, but now we focus on EtEQ instead of LQ. To the best of our knowledge, this is the first attempt to predict EtEQ in WMCN.

\section{AnAlysis of Results}

\section{A. Experimental Framework}

As stated before, we deal with time series analysis to estimate link quality in the routing layer for real-world WMCN. To do this we assume the FunkFeuer experimental network and an OLSR data set of several nodes and links.

A time series is a set of data collected over time with a natural temporal ordering. It differs from typical data mining or machine learning applications, where the ordering of data points within a data set is not important. Time series analysis is the process of using statistical techniques to model and explain a time-dependent series of data points. Similarly, time series forecasting is a method that uses a model to generate predictions (forecasts) of future events based on known past events. In our case, we used more than one prediction algorithm so that we do not rely on a specific learning technique. We applied four of the best well-known approaches [34]: Support Vector Machines (SVM), k-Nearest Neighbors (kNN), Regression Trees (RT) and Rule-Based Regression (RBR).

The Support Vector Machines (SVM) algorithm has recently become one of the most popular and widely used methods in machine learning. It performs a linear or nonlinear division of the input space and builds a prediction model that assigns target values into one or another category. The kNearest Neighbors $(\mathrm{kNN})$ algorithm is one of the most simple machine learning algorithms as it makes no assumptions on the underlying data distribution. This algorithm takes the $\mathrm{k}$ data-points closest to the target value and picks the most common one. Regression Tree (RT) is a type of decision tree algorithm where the target value can have continuous values. This method recursively partitions the data space and runs a simple prediction model within each partition. Finally, the Rule-Based Regression (RBR) algorithm is similar to a 


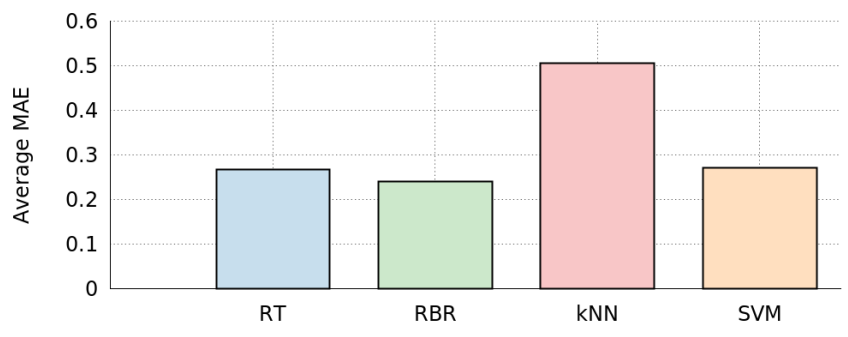

Figure 6. Average Mean Absolute Error (MAE) of the paths.

decision tree approach but it is a stronger model that provides rules that are often potentially more predictive

We applied training and test sets validation to evaluate the predictive accuracy of the models. After a model is processed using the training set, it is tested by making predictions against the test set. For this purpose, we used the Weka workbench system [35], a framework that incorporates a variety of learning algorithms and some tools for the evaluation and comparison of the results. Weka has a dedicated environment for time series analysis that allows forecasting models to be developed and evaluated. The Weka's time series framework takes a machine learning or data mining approach to model time series by transforming the data into a form that can be processed by standard propositional learning algorithms. To do so, it removes the temporal ordering of individual inputs by encoding the time dependency via additional input fields.

Usually, classification studies assess the predictive power of their model by using Mean Absolute Error (MAE) or Root Mean Squared Error (RMSE), both widely used in related work. We assume MAE in our experiments as it is a common method to evaluate the performance of prediction approaches, that gives the same weight to all individual differences. This metric is calculated through the following formula: $M A E=$ $\operatorname{sum}($ abs $($ predicted - actual $)) / N$.

\section{B. Comparison of learning algorithms based on time series}

As stated before, we want to explore whether time series analysis can be used to predict future End-to-End quality values. To do this, we applied four well-known approaches: Support Vector Machines (SVM), k-Nearest Neighbors (kNN), Regression Trees (RT) and Rule-Based Regression (RBR).

Figure 6 shows the average Mean Absolute Error (MAE) per path using a training data set of 2016 instances (7 days), a test data set of 288 instances (1 day) and a lag window composed of the last 12 instances. This test was performed to verify whether time series learning algorithms could predict consecutive EtEQ values. These results show that we achieved the best accuracy for the Rule-Based Regression (RBR) and the worst for k-Nearest Neighbors (kNN). Regression Trees (RT) and Support Vector Machines (SVM) also moves very close to RBR results. Notice that the maximum EtEQ value is 1 and therefore, the MAE per link is $2.4 \%$ for $\mathrm{RBR}$ and $5 \%$ for $\mathrm{kNN}$. We applied a T-test to mean values for independent samples (at 95\% confidence level) in order to compare the classification algorithms using the MAE. After this analysis, p-values smaller than 0.05 indicate that the means are significantly different, and therefore, we would

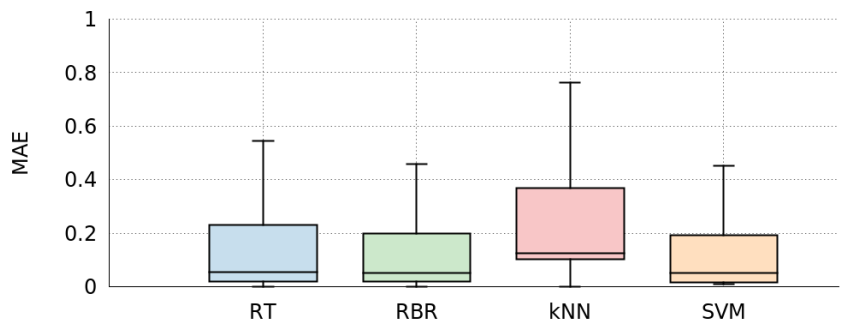

Figure 7. Mean Absolute Error (MAE) of the EtEQ predictions as boxplot.

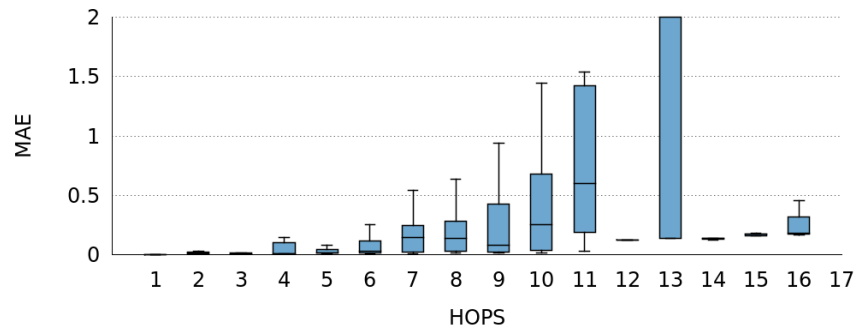

Figure 8. Distribution of RBR Mean Absolute Error (MAE) as boxplot.

reject the null hypothesis of no difference between the means. Consequently, we can claim that time-series analysis achieves high percentages of success and that among them, RBR seems to be the best candidate to make predictions.

We also analyzed the error variability of each algorithm and represented the results using boxplots. Three of the four algorithms achieved similar performance for most of the links (RT, RBR and SVM), as shown in Figure 7. Although, RT may present some outliers, the differences among median, 1st quartile and 3rd quartile are minimal. On the other side, kNN presents different behavior compared to the others. In this case outliers present larger errors that increase the average values and change the overall evaluation of the algorithm. The rest of the paper, we assume RBR algorithm to show the potential benefits of predicting EtEQ by means of a time series analysis.

\section{EtEQ Prediction with Rule-Based Regression}

We proceed next to study more in depth the EtEQ using the RBR algorithm, in order to discover how can we reach a satisfactory level of prediction.

Figure 8 presents boxplots of the MAE of path ETX prediction with RBR versus the number of Hops corresponding to the paths. We used the same training data set as section V.B. Even though the dispersion of the error seems high in specific cases (for example 10,11 and 13 hops), the results follow the dispersion pattern of actual ETX values as described in figure 5 , though in a much smaller scale. In other words, even though ETX values for 10, 11 and 13 hop paths have a high dispersion, our prediction manages to successfully predict a big percentage of the fluctuations. For instance, the dispersion of 13 hop path ETX is 6, while the error of its prediction has a maximum value of 3. For the rest of the paths, the MAE has maximum value less than 1 , resulting to a meaningful prediction.

Figure 9 provides a more detailed analysis of the prediction accuracy. We can see that the average ETX value and the 


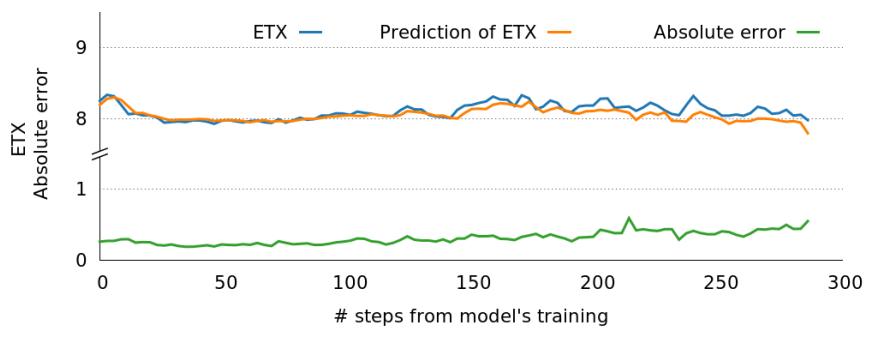

Figure 9. Evolution of the average ETX, the average prediction and the average absolute error.
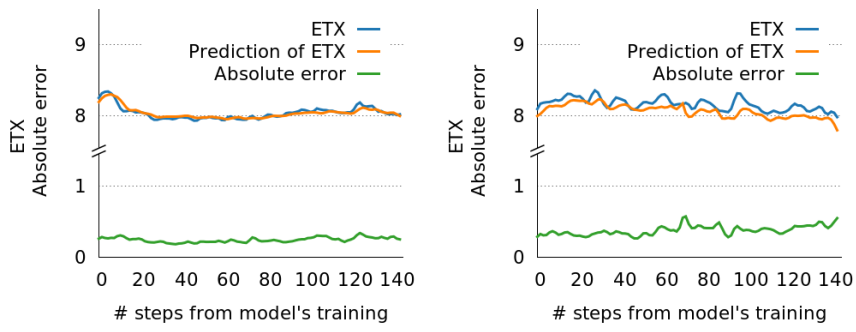

Figure 10. Evolution of the average ETX, the average prediction and the average absolute error for: a) $12 \mathrm{am}-12 \mathrm{pm}$, b) $12 \mathrm{pm}-12 \mathrm{am}$

average prediction value are very close, even overlapping during the first half of prediction test. A better estimation for the deviation of the individual path values is given by the average absolute error line. Notice that the deviation remains less than 0.5 throughout the whole prediction, which is a great achievement. Nevertheless, the potential impact of this small error in routing decisions can be further studied.

Another characteristic of the prediction revealed by Figure 9 is that after 100 steps of prediction (between 8 a.m. and 8:30 a.m.) the absolute error presents an increasing trend. We assume that this outcome is an effect of the actual ETX oscillations depicted in Figure 3 and not the result of a possible degradation by time of the prediction accuracy. In order to verify this assumption we performed two more prediction tests. From 12 a.m. to 12 p.m. (Figure 10.a) and from 12 p.m. to 12 a.m. (figure 10.b), using as training data set the 2016 more recent instances (7 days before prediction starts), as test data set 144 instances (half a day) and a lag window composed of the last 12 instances. The results obtained are almost identical to the results of Figure 9, leading to the conclusion that the ETX oscillations are indeed affecting the prediction. Therefore, we plan to explore in the future how accuracy could be increased by deploying two different predictors, for day and night.

\section{Prediction of Some Steps Ahead}

This analysis was performed to explore if time series analysis and prediction can be used to predict the value of EtEQ some time steps ahead into the future.

Figure 11 shows the average MAE of paths. It shows the results of the RBR algorithm using the same setup that the baseline experiment (a lag window size of 12 instances, a training dataset of 2016 instances and a test dataset of 288 instances) and then predicting from 1 to 10 time steps into the

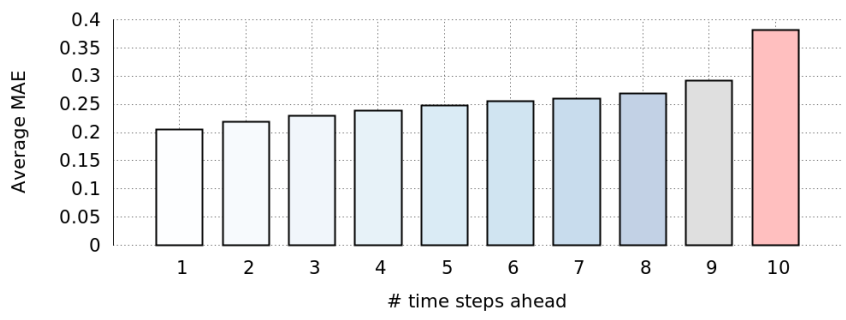

Figure 11. RBR average Mean Absolute Error (MAE) of the EtEQ predictions.

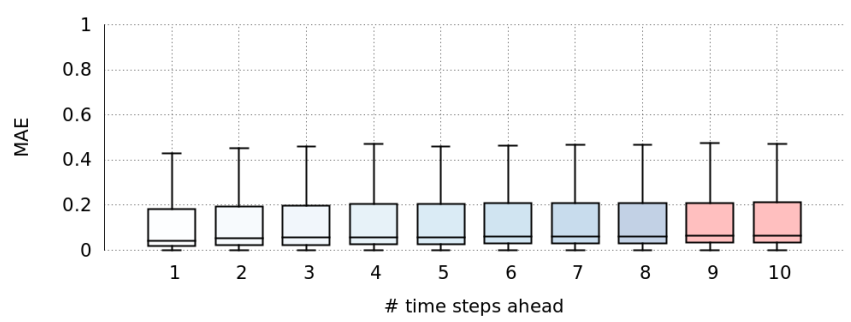

Figure 12. RBR Mean Absolute Error of EtEQ predictions, depicted as a boxplot.

future. The results obtained were good for the majority of the tests. As we can observe, the average MAE grows very slowly. It seems possible to affirm that we could predict successfully the EtEQ several steps ahead in time.

Once more, we analyzed the variability of errors for each number of steps ahead using a box plot, shown in Figure 12. Although the values for the median and the first quartile are similar for all steps ahead considered, the values of third quartile and outliers (no depicted) grow with the number of steps. These differences in the variability of errors lead to the differences in the average MAE.

\section{CONCLUSIONS}

This study demonstrates that time series analysis is a promising approach to accurately predict EtEQ values in community networks. This technique can be used to improve the performance of the routing protocol by providing information to make appropriate and timely decisions to maximize the delivery rate and minimize traffic congestion.

The dataset used in our work shows quite significant fluctuations (about 7\%) in the temporal evolution of the number of paths (routing entries). This contrasts with the general assumption in mesh networks that the number of paths is stable. The persistence of paths in our dataset is very stable for $90 \%$ of paths, but there are $10 \%$ of paths with persistence values below $60 \%$, and those paths create the temporal fluctuations in the number of paths. Our study includes paths with persistence above $25 \%$. Regarding the ETX behavior, the average ETX path quality is more stable at night, and presents more fluctuations during the morning. In non-working days, this behavior is less apparent. We assume this is due to the variation of network traffic and interference during the day, leading to packet loss. Hence, the accuracy of prediction depends on the day and time when it is applied. On 
the other side, the most frequent number of Hops and ETX values are in the range from 5 to 10 . The dispersion of ETX according to the number of Hops is minimal between 1 and 4 Hops, and for more than 14 Hops. However, the dispersion is significant between 5 and 9 Hops and relevant for 10 to 13 Hops. Therefore, the accuracy of the predictions would be better or worse depending on the number of Hops.

We have presented results from four well known learning algorithms that model time series. All of them achieved high percentages of success, with average Mean Absolute Error values per link between $2.4 \%$ and $5 \%$ when predicting the next value of the EtEQ. We also analyzed the error variability and found that three of them presented similar performance (RT, RBR and SVM), whereas kNN performs worse due to outliers with larger errors. A more detailed study of RBR prediction shows an average absolute error less than 1 . We have also observed differences in the prediction behavior during day and during night, as it happens with actual ETX values.

As future work, we want to extend this analysis to other community networks to evaluate if the observed behaviour could be generalized. Moreover, we plan to identify which paths contribute most to the errors in the EtEQ prediction and to understand what factors make it more difficult to predict them. We also want to study the impact of errors in routing decisions, and study a solution with two different predictors for day and night. Finally, we plan to improve the prediction process discarding those paths whose relation between EtEQ and prediction accuracy is above a certain threshold.

\section{ACKNOWLEDGMENTS}

This work was partially supported by the European Community through the projects Community Networks Testbed for the Future Internet (CONFINE): FP7-288535, and A Community networking Cloud in a box (Clommunity): FP7-317879, and also by Spanish government under contract TIN201347245-C2-1-R, and also by the Generalitat de Catalunya as a Consolidated Research Group 2014-SGR-881.

\section{REFERENCES}

[1] J. Avonts, B. Braem and C. Blondia. "A Questionnaire Based Examination of Community Networks". International Workshop on Community Networks and Bottom-up-Broadband, 2013

[2] FunkFeuer, a free, experimental network in Vienna. http://www.funkfeuer.at/

[3] Open, Free and Neutral Network Internet for everybody. http://guifi.net/

[4] B. Braem et al, "A Case for Research with and on Community Networks". Computer Communication Review, vol.43, 2013

[5] A. Woo, et al. "Taming the Underlying Challenges of Reliable Multihop Routing in Sensor Networks". International Conference on Embedded Networked Sensor Systems, 2003.

[6] R. Draves, J. Padhye and B. Zill. "Comparison of Routing Metrics for Static Multi-Hop Wireless Networks". the ACM Sigcomm, vol.34, 2004.

[7] C. E. Koksal and H. Balakrishnan. "Quality-Aware Routing Metrics for Time-Varying Wireless Mesh Networks". Journal on Selected Areas in Communications, vol.24, 2006

[8] T. Rault, A. Bouabdallah and Y. Challal. "Energy Efficiency in Wireless Sensor Networks: A Top-Down Survey”. Journal of Computer Networks, vol 67, 2014.

[9] J. F. Rodríguez-Covili et al, "A Communication Infrastructure to Ease the Development of Mobile Collaborative Applications". Journal of Network and Computer Applications, vol.34, 2011.
[10] S. Vural, Dali Wei, and K. Moessner. "Survey of Experimental Evaluation Studies for Wireless Mesh Network Deployments in Urban Areas Towards Ubiquitous Internet”. Comm. Surveys Tutorials, vol.15, 2013.

[11] N. Baccour, et al. "Radio Link Quality Estimation in Wireless Sensor Networks: a Survey", Transactions on Sensor Networks, vol.8, 2012.

[12] L. Lu and T. Zhou. "Link Prediction in Complex Networks: A Survey", Physica A: Statistical Mechanics and its Applications, vol.390, 2011.

[13] P. Millán, C. Molina, R. Meseguer, E. Medina, D. Vega, B. Braem, C Blondia, "Tracking and Predicting Link Quality in Wireless Community Networks", In Procs of the 10th International Conference on Wireless and Mobile Computing, Networking and Communications, 2014.

[14] FunkFeuer-CONFINE Open Data. http://opendata.confineproject.eu/group/funkfeuer

[15] D. De Couto et al, "A High Throughput Path Metric for Multi-Hop Wireless Routing". Mobicom Conf, 2003.

[16] R. Meseguer, C. Molina, S. F. Ochoa, R. Santos, "Energy-Aware Topology Control Strategy for Human-Centric Wireless Sensor Networks". Sensors Journal, vol.14, 2014.

[17] R. Meseguer, C. Molina, S. F. Ochoa, R. Santos. "Reducing Energy Consumption in Human-Centric Wireless Sensor Networks". International Conference on Systems, Man, and Cybernetics, 2012.

[18] J. Harri, F. Filali and C. Bonnet. "Kinetic Multipoint Relaying: Improvements Using Mobility Predictions". In Active and Programmable Networks, LNCS, vol. 4388, 2009.

[19] M. Maleki, K. Dantu and M. Pedram, "Lifetime Prediction Routing in Mobile Ad Hoc Networks", Wireless Comm. \& Networking, 2003.

[20] D. Kim, J. J. Garcia-Luna-Aceves and K. Obraczka. "Routing Mechanisms for Mobile Ad Hoc Networks Based on the Energy Drain Rate". IEEE Transactions on Mobile Computing vol.2, 2003.

[21] Y. Luo, J. Wang and S. Chen. "An Energy-Efficient DSR Routing Protocol Based on Mobility Prediction". International Symposium on a World of Wireless, Mobile and Multimedia Networks, 2006.

[22] R. Fonseca et al. "Four Bit Wireless Link Estimation". Int. Workshop on Hot Topics in Networks, 2007.

[23] M. Yunquian. "Improving Wireless Link Delivery Ratio Classification with Packet SNR". International Conference on Electro Information Technology, 2005.

[24] M. Senel, et al. "A Kalman Filter Based Link Quality Estimation Scheme for Wireless Sensor Networks". Global Comm. Conf, 2007.

[25] A. Cerpa et al. "Temporal Properties of Low Power Wireless Links: Modeling and Implications on Multi-Hop Routing". International Symposium on Mobile Ad Hoc Networking and Computing, 2005.

[26] S. Naimi et al, "Anticipation of ETX Metric to Manage Mobility in Ad Hoc Wireless Networks", International Conf. ADHOC-NOW, 2014.

[27] C. Renner et al, "Prediction Accuracy of Link-Quality Estimators". European Conference of Wireless Sensor Networks, 2011.

[28] D. Passos and C. V. N. Albuquerque, "A Joint Approach to Routing Metrics and Rate Adaptation in Wireless Mesh Networks", Journal IEEE/ACM Transactions on Networking, 2012.

[29] S. Banerjee and A. Misra, "Minimum Energy Paths for Reliable Communication in Multi-hop Wireless Networks", In Procs of the 3rd Int. Symposium on Mobile ad Hoc Networking \& Computing, 2002.

[30] G. Jakllari et al, "Link Positions Matter: A Noncommutative Routing Metric for Wireless Mesh Network", In Procs of the IEEE 27th Conference on Computer Communications, 2008.

[31] H. Li et al, "Minimizing End-to-End Delay: A Novel Routing Metric for Multi-Radio Wireless Mesh Networks", In Procs of the 28th Conference on Computer Communications, 2009.

[32] Y. Wang, et al. "A New Scheme on Link Quality Prediction and its Applications to Metric-Based Routing". International Conference on Embedded Networked Sensor Systems, 2005.

[33] L. Maccari, R. L. Cigno, "A Week in the Life of Three Large Wireless Community Networks", Ad Hoc Networks vol. 24, 2015

[34] M. A. Alsheikh et al. "Machine Learning in Wireless Sensor Networks: Algorithms, Strategies, and Applications". The IEEE Communications Surveys \& Tutorials, vol.16, 2014.

[35] M. Hall, et al. "The WEKA Data Mining Software: An Update". SIGKDD Explorations, vol.11, 2009. 